\title{
Invasive Pulsed-Type, Bipolar, Alternating Current Radiofrequency Treatment Using Microneedle Electrodes for Nasal Rosacea
}

Tae Hwan Ahn' ${ }^{1}$

Sung Bin $\mathrm{Cho}^{2,3}$

${ }^{1}$ Fresh Facial Aesthetic Surgery and ENT Clinic, Seoul, Korea

${ }^{2}$ Department of Dermatology and Cutaneous Biology Research Center, International St. Mary's Hospital, Catholic Kwandong University College of Medicine, Incheon, Korea

${ }^{3}$ Kangskin Sillim Dermatology Clinic, Seoul, Korea
Received June 9, 2017

Accepted June 12, 2017

\section{Correspondence}

Sung Bin Cho

Department of Dermatology and Cutaneous

Biology Research Center, International St. Mary's Hospital, Catholic Kwandong University College of Medicine, 25 Simgok-ro, Seo-gu, Incheon 22711, Korea

Tel.: +82-32-290-3141

Fax: +82-32-290-3142

E-mail: drsbchođggmail.com

(c) Korean Society for Laser Medicine and Surgery

(c) This is an open access article distributed under the terms of the Creative Commons Attribution NonCommercial License (http://creativecommons.org/ licenses/by-nc/4.0) which permits unrestricted noncommercial use, distribution, and reproduction in any medium, provided the original work is properly cited.
Radiofrequency (RF) devices deliver high frequency energy to the biological tissues thath as been either cut or coagulated. A pulsed-type RF irradiation elicits thermal and/or non-thermal effects by delivering a particular rate of gated RF oscillations. In the present study, we describe two patients with nasal rosacea, who were effectively treated with invasive pulsed-type, bipolar alternating current (AC) RF treatment using microneedle electrodes. The RF treatment was delivered to these two patients with a power level of 3 , a microneedle penetration depth of 1.5 $\mathrm{mm}$, and 2-3 passes over six (patient 1) and 12 (patient 2) sessions, at two-week intervals. The first patient presented marked clinical improvement with respect to both the overall redness and papulopustular lesions; the second patient exhibited noticeable improvement in both the texture and telangiectatic lesions on the nose. Both patients were satisfied with the clinical outcomes without remarkable side effects.

\section{Key words}

Pulsed-type radiofrequency; Bipolar; Alternating current; Invasive; Nose; Rosacea; Telangiectasia 


\section{INTRODUCTION}

Rosacea is a chronic inflammatory disorder of the facial and extrafacial skin that clinically appears as flushing and blushing, persistent erythema and telangiectasia, persistent plaque-form edema, and/or acneiform papulopustules and nodules. ${ }^{1,2}$ The five subtypes of rosacea, according to the modified National Rosacea Society classification system, include erythematotelangiectatic, papulopustular, phymatous, ocular, and mixed subtypes. ${ }^{3-5}$ Among Koreans with localized nasal rosacea, erythematotelangiectatic rosacea is the most common subtype, followed by papulopustular rosacea. ${ }^{3}$ Compared with phymatous or mixed subtypes, erythematotelangiectatic rosacea localized to the nasal areas exhibits shorter disease duration and lower severity. ${ }^{3}$

Various energy delivery devices, including pulsed-dye laser and intense pulsed light, have been used for the treatment of rosacea as alternative or combination treatment modalities. ${ }^{2}$ Long-pulsed lasers with a pulse duration of 0.35 to 40 milliseconds at the wavelengths of 585 $\mathrm{nm}$ or $595 \mathrm{~nm}$ effectively destroy congenital and acquired vascular lesions through the selective photothermolysis of target chromophores. ${ }^{6}$ The laser energy is preferentially absorbed by vascular components with a sufficient number of chromophores. Thus, pulsed-dye laser therapy has been suggested as an additional therapeutic option for improving rosacea with grade $\mathrm{C}$ and $\mathrm{D}$ recommendations. ${ }^{6}$ In the present study, we report the cases of two patients with papulopustular or erythematotelangiectatic nasal rosacea who were effectively treated with an invasive pulsed-type, bipolar alternating current (AC) radiofrequency (RF) device using microneedle electrodes.

\section{CASE REPORT}

Two Korean male patients (24-year-old and 76-yearold) with Fitzpatrick skin type IV, who were clinically diagnosed with nasal rosacea, participated in this study. The first patient presented with localized, recurrent erythematous papulopustular lesions on the nose, along with a few pustules on the perioral area (Fig. 1A). The second patient
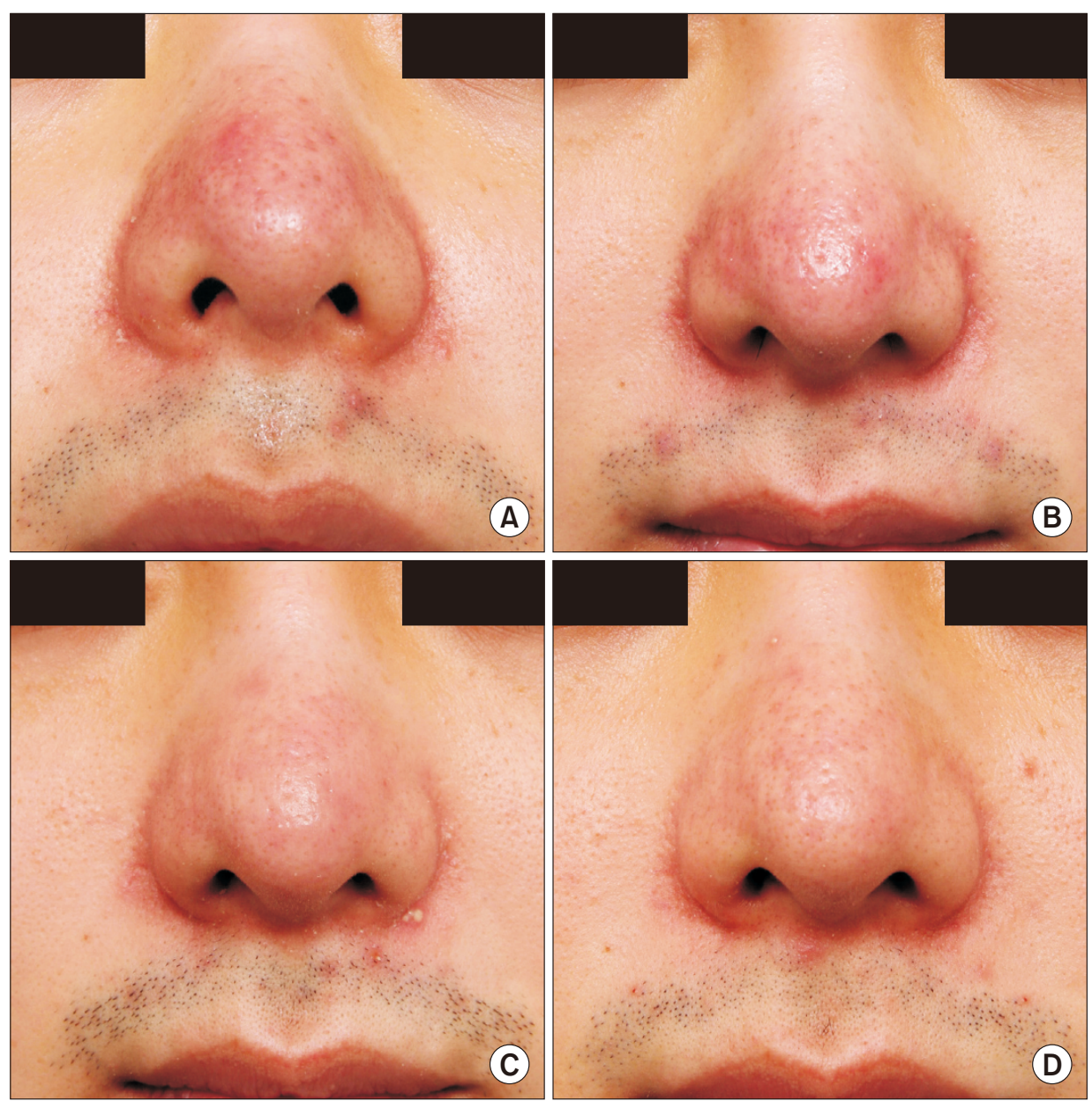

Fig. 1. Normal light-exposed photos reveal papulopustular rosacea along the nose in a 24-year old man at (A) baseline, at two weeks after (B) two sessions and (C) four sessions, and (D) at four weeks after six sessions of invasive pulsed-type, bipolar alternating current $(\mathrm{AC})$ radiofrequency $(\mathrm{RF})$ treatment using microneedle electrodes. 
exhibited markedly telangiectatic lesions on a mildly phymatous nose (Fig. 2A). Previously, the first patient had been intermittently treated with oral and topical antibiotics for inflammatory acne lesions; neither patients had been treated with any light or laser devices, oral or topical retinoids, or topical metronidazole or brimonidine tartrate gels for the treatment of localized nasal rosacea within the most recent 1 year. Otherwise, they had no remarkable familial or medical history.

After obtaining written informed consent, the patients were treated with six (patient 1) and 12 (patient 2) sessions of invasive pulsed-type, bipolar AC RF treatment using microneedle electrodes (SYLFIRM; Viol, Kyunggi, Korea) for localized nasal rosacea at two-week intervals. The skin along the nose was cleansed with 70\% ethanol and anesthetized with topical anesthetic cream for 30 minutes prior to the treatment. Then, invasive pulsed-type, bipolar AC RF treatment was delivered with a power level of 3 , a microneedle penetration depth of $1.5 \mathrm{~mm}$, and 2-3 passes. All shots were delivered after gently pressing the disposable tips on the skin for proper penetration of the microneedle electrodes into the target area. Immediately after treatment, the treatment area was cooled with icepacks. No prophylactic systemic or topical antibiotics and corticosteroids were prescribed to the patients immediately after or during the course of treatment.

The first patient presented with marked clinical improvement in both overall redness and the papulopustular lesions on the nose from the fourth session of treatment with the invasive pulsed-type, bipolar AC RF device. Further improvement in the nasal rosacea was recorded with subsequent treatments (Fig. 1B-D). The second patient experienced noticeable improvement in both the texture and the telangiectatic lesions of the nose from the fifth session of treatment, and pronounced clinical improvement was recorded at one month after the final treatment session (Fig. 2B-D). Both of the patients were very satisfied with the clinical outcomes, and no remarkable side effects, including immediate excessive bleeding or oozing, worsening of the nasal rosacea, folliculitis or furuncle, post-therapy prolonged erythema, post-therapy dyschromia, or scarring, were reported. As well, pain during the

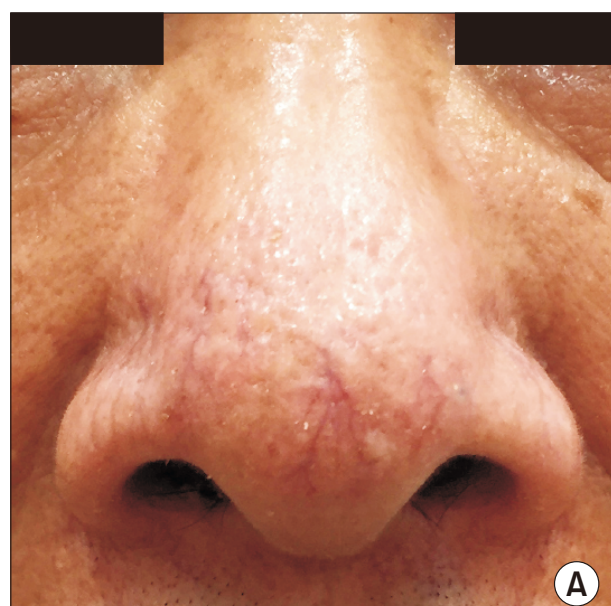

(A)

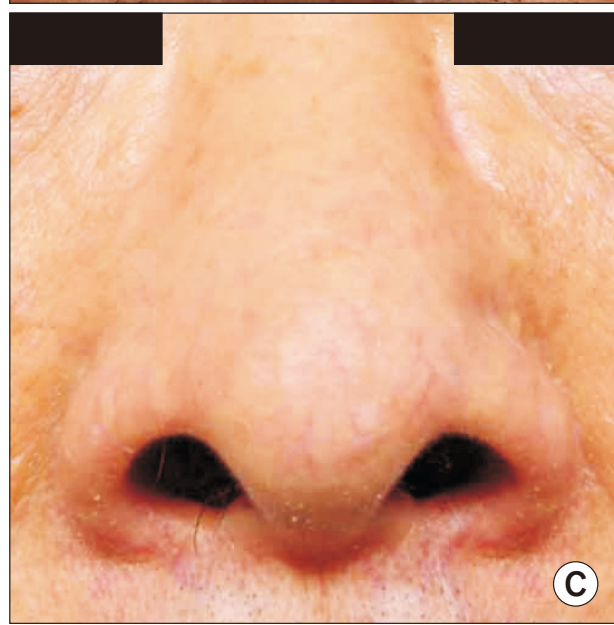

(C)

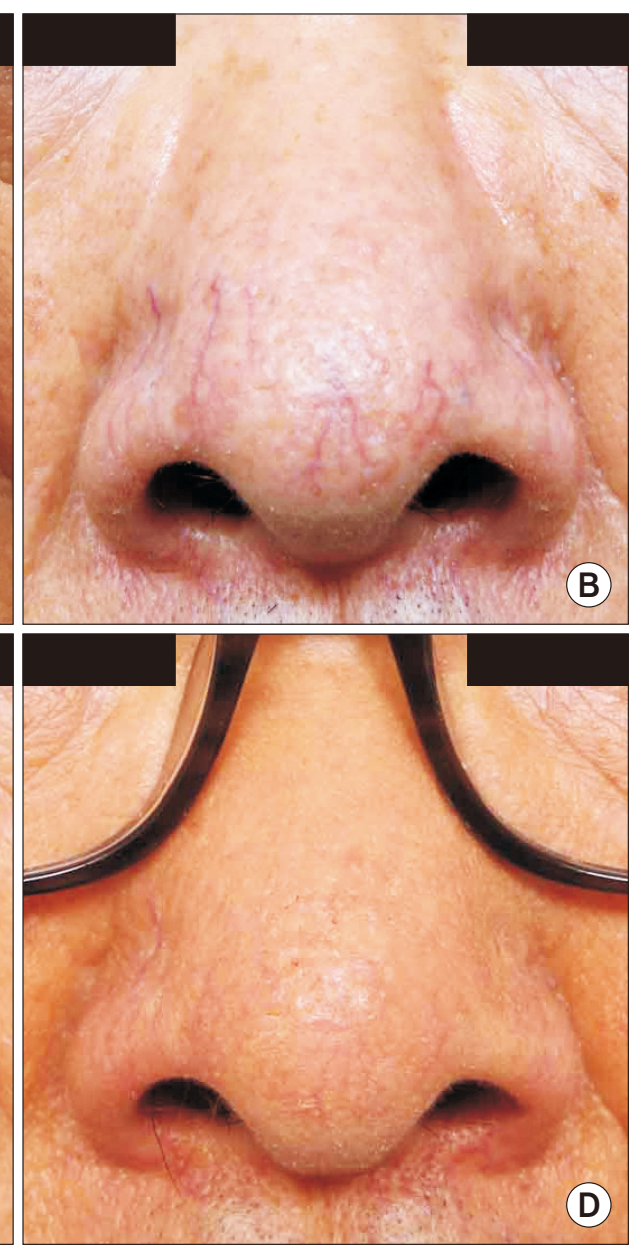

Fig. 2. Normal light-exposed photos reveal erythematotelangiectatic nasal rosacea in a 76-year old man at (A) baseline, at two weeks after (B) two sessions and (C) seven sessions, and (D) at four weeks after 12 sessions of invasive pulsed-type, bipolar AC RF treatment using microneedle electrodes. 
invasive pulsed-type, bipolar AC RF treatment was well tolerated with topical anesthetic cream in both patients.

\section{DISCUSSION}

RF devices deliver high frequency (HF) monopolar or bipolar AC to the skin in both noninvasive and minimally invasive manners. ${ }^{7}$ Invasive bipolar AC RF can deliver electrical currents deeper to target tissues by penetrating microneedle electrodes into the skin, and the penetration depth of HF energy is relatively predictable, compared to monopolar RF ${ }^{7,8}$ Additionally, continuous-type RF irradiation generates thermal ablative effects in target tissues by continuously delivering HF energy over set conduction times, whereas pulsed-type RF irradiation elicits thermal and/or non-thermal effects by delivering a particular rate of gated RF oscillations. ${ }^{7,9}$ With these characteristics, invasive pulsed-type, bipolar AC RF treatment has been deemed effective for use in treating refractory melasma. ${ }^{10}$

The skin exhibits various current densities among its multiple layers and also among different types of appendage structures. Thus, the patterns of invasive, continuoustype, bipolar, AC RF-induced skin reactions differ mainly according to the depth of microneedle penetration, RF conduction time, and signal amplitude. ${ }^{11,12}$ Regardless of the use of insulated or non-insulated microneedles, a larger area of tissue coagulation is generated from the tips of individual microneedles by increasing the depth of penetration or RF conduction time, while a higher signal amplitude induces a higher degree of tissue coagulation. ${ }^{7,11,12} \mathrm{~A}$ previous in vivo micropig skin study revealed that tissue coagulation induced by continuous-type, bipolar AC RF is found preferentially along the outer layers of vessels and perivascular structures in the dermis between individual non-insulated microneedle electrodes. ${ }^{12}$

Our two patients presented with localized papulopustular and erythematotelangiectatic nasal rosacea, respectively. As the vascular components and surrounding dermal tissues exhibit high current densities, the pulsedtype delivery of bipolar RF energy was able to effectively treat the abnormal vascular components of the nasal rosacea lesions in our patients without producing excessive thermal injury to the epidermis and dermis. However, the optimal treatment settings for maximizing the vascular selectivity of invasive pulsed-type, bipolar AC RF energy remains to be elucidated.

Both of the patients demonstrated noticeable clinical improvement after four to five sessions of invasive pulsed-type, bipolar AC RF treatment and showed further improvement after additional sessions of the treatment.
Interestingly, the first patient also experienced marked decreases in inflammation, and the other patient showed textural improvement in the phymatous-appearance of his nose. This led us to suggest that our treatment using a pulsed-type RF device could have generated subcellular, electrical thermo-modulating effects on the inflammatory cells and dermal mesenchymal cells, in addition to the dermal vascular components.

In this report, invasive pulsed-type, bipolar AC RF treatment using non-insulated microneedle electrodes proved to be safe and effective in treating papulopustular and erythematotelangiectatic rosacea localized to the nose in two male Korean patients. Accordingly, we suggest that treatment with invasive pulsed-type, bipolar AC RF devices may be an additional therapeutic option for treating various types of localized nasal rosacea. However, histopathologic investigations to evaluate the effects thereof on targeted vascular components are lacking. Randomized, prospective, controlled clinical trials should be followed to confirm our findings.

\section{ACKNOWLEDGEMENTS}

We would like to thank Anthony Thomas Milliken, ELS, (Editing Synthase, Seoul, Korea) for his help with the editing of this manuscript.

\section{REFERENCES}

1. Plewig G, Kligman AM. Acne and rosacea. 3rd ed. Berlin: Springer; 2000. p.455-502.

2. Bernstein EF, Kligman A. Rosacea treatment using the newgeneration, high-energy, $595 \mathrm{~nm}$, long pulse-duration pulseddye laser. Lasers Surg Med 2008;40:233-9.

3. Lee WJ, Lee YJ, Won CH, Chang SE, Choi JH, Lee MW. Clinical evaluation of 30 patients with localized nasal rosacea. J Dermatol 2016:43:200-2.

4. Wilkin J, Dahl M, Detmar M, Drake L, Feinstein A, Odom R, et al. Standard classification of rosacea: report of the national rosacea society expert committee on the classification and staging of rosacea. J Am Acad Dermatol 2002;46:584-7.

5. Wilkin J, Dahl M, Detmar M, Drake L, Liang MH, Odom R, et al. Standard grading system for rosacea: report of the national rosacea society expert committee on the classification and staging of rosacea. J Am Acad Dermatol 2004;50:907-12.

6. Erceg A, de Jong EM, van de Kerkhof PC, Seyger MM. The efficacy of pulsed dye laser treatment for inflammatory skin diseases: a systematic review. J Am Acad Dermatol 2013;69:609-15.e8.

7. Cho SB, Kim HS. High-frequency alternating electrical 
current: selective electromagnetic tissue reaction. Med Lasers 2016:5:1-6.

8. Taheri A, Mansoori P, Sandoval LF, Feldman SR, Pearce D, Williford PM. Electrosurgery: part I. Basics and principles. J Am Acad Dermatol 2014;70:591.e1-14.

9. Yarmush ML, Golberg A, Serša G, Kotnik T, Miklavčič D. Electroporation-based technologies for medicine: principles, applications, and challenges. Annu Rev Biomed Eng 2014;16:295-320.

10. Choi M, Choi S, Kang JS, Cho SB. Successful treatment of refractory melasma using invasive micro-pulsed electric signal device. Med Lasers 2015;4:39-44.

11. Zheng Z, Goo B, Kim DY, Kang JS, Cho SB. Histometric analysis of skin-radiofrequency interaction using a fractionated microneedle delivery system. Dermatol Surg 2014;40:134-41.

12. Na J, Zheng Z, Dannaker C, Lee SE, Kang JS, Cho SB. Electromagnetic initiation and propagation of bipolar radiofrequency tissue reactions via invasive non-insulated microneedle electrodes. Sci Rep 2015;5:16735. 